\title{
Real time magnetic control of the snowflake plasma configuration in the TCV tokamak
}

To cite this article before publication: Himank Anand et al 2019 Nucl. Fusion in press https://doi.org/10.1088/1741-4326/ab4440

\section{Manuscript version: Accepted Manuscript}

Accepted Manuscript is "the version of the article accepted for publication including all changes made as a result of the peer review process, and which may also include the addition to the article by IOP Publishing of a header, an article ID, a cover sheet and/or an 'Accepted

Manuscript' watermark, but excluding any other editing, typesetting or other changes made by IOP Publishing and/or its licensors"

This Accepted Manuscript is @ EURATOM 2019.

During the embargo period (the 12 month period from the publication of the Version of Record of this article), the Accepted Manuscript is fully protected by copyright and cannot be reused or reposted elsewhere.

As the Version of Record of this article is going to be / has been published on a subscription basis, this Accepted Manuscript is available for reuse under a CC BY-NC-ND 3.0 licence after the 12 month embargo period.

After the embargo period, everyone is permitted to use copy and redistribute this article for non-commercial purposes only, provided that they adhere to all the terms of the licence https://creativecommons.org/licences/by-nc-nd/3.0

Although reasonable endeavours have been taken to obtain all necessary permissions from third parties to include their copyrighted content within this article, their full citation and copyright line may not be present in this Accepted Manuscript version. Before using any content from this article, please refer to the Version of Record on IOPscience once published for full citation and copyright details, as permissions will likely be required. All third party content is fully copyright protected, unless specifically stated otherwise in the figure caption in the Version of Record.

View the article online for updates and enhancements. 


\title{
Real time magnetic control of the snowflake plasma configuration in the TCV tokamak
}

\author{
H. Anand ${ }^{1}$, S. Coda ${ }^{1}$, F. Felici ${ }^{1}$, C. Galperti ${ }^{1}$, J.- M. Moret ${ }^{1}$, \\ B. Labit ${ }^{1}$, H. Reimerdes ${ }^{1}$, R. Maurizio ${ }^{1}$, and the TCV team ${ }^{1}$ \\ ${ }^{1}$ École Polytechnique Fédérale de Lausanne (EPFL), Swiss Plasma \\ Center (SPC), EPFL SB SPC, Station 13, CH-1015 Lausanne, \\ Switzerland.
}

August 19, 2019

\begin{abstract}
The novel plasma position and shape controller of the TCV tokamak aids in the precise control of complex configurations such as snowflake (SF) divertors. The ability of the controller design to simultaneously control the position, shape, divertor leg and X-points is extended to various SF plasma configurations. The unique feature of ordering the controlled variables from the most easily to the least easily controlled, while respecting the hardware limits on the poloidal-field coil currents, is exploited in particular to provide reliable control of SF equilibria with closely spaced X-points, approaching the exact snowflake. The off-line optimisation of the control parameters and their experimental implementation on snowflake plasma configuration is reported. The performance of the new controller is compared with the legacy TCV analogue controller for a SF plasma discharge. The new controller is successful in providing a marked improvement over the legacy TCV controller with respect to $\sigma$, the main parameter of interest for this study.
\end{abstract}

\section{Introduction}

The nested magnetic flux surfaces necessary for plasma confinement are obtained in a tokamak by the combination of fields produced by the external conductors and the plasma itself. The shape of the plasma is determined by the last closed flux surface. This is defined either by the intersection of the closed magnetic surfaces with a solid surface (limited plasma) or by a singularity in the magnetic field itself. The latter geometry, known as divertor configuration, has emerged 
as the pre-eminent solution for managing power and particle exhaust and core impurity content. At the magnetic X-point, a saddle point in the magnetic flux distribution, the poloidal field vanishes, and the topology allows energy and particle losses to be channeled primarily into the divertor region that is separated from the confined plasma region. The downside is the high peak heat flux on the divertor plates, which can lead to destructive overheating and erosion.

In the ITER tokamak $Q=10$ scenario, the steady-state power that crosses the separatrix and enters into the scrape-off layer (SOL) is expected to be about 100 MW. The distribution of the power has to be optimised such that the peak heat fluxes on the plasma facing components are below the maximum tolerable value of $15 \mathrm{MW} / \mathrm{m}^{2}$. The requirements for power handling in ITER can be fulfilled provided $60-70 \%$ of the power entering the SOL is exhausted through radiation in order to spread the power over a larger area 11. Moreover the radiation fractions [2, 3] in future fusion reactors including DEMO must even be higher [4]. Approaches to cope with the heat fluxes expected in DEMO include the development of new materials that can withstand greater steadystate heat fluxes [5], techniques and operating regimes that allow for a higher value of radiation loss [6] and techniques to increase the wetted area. Alternative divertor concepts are being considered to control the heat loads on the divertor targets and are currently under intense investigation. Different solutions have been proposed to reduce the plasma-wall interaction in the divertor region by acting on the magnetic field topology [7, 8, 9, 10, 11]. One of these solutions is the so-called snowflake (SF) divertor [7].

A conventional single-null (SN) divertor configuration is formed by cancelling the poloidal magnetic field $B_{\theta}$ at the null point, which can be achieved with a dedicated divertor coil as shown in Figure 1(a). In the SF configuration, which requires additional divertor coils, the gradient of the poloidal field at the nullpoint also vanishes $\left(\nabla B_{\theta}=0\right), \mathbb{1}(\mathrm{b})$, thereby creating a second order null-point.

In reality, an exact SF configuration, Figure 2(a), is only a single point in an operational domain and, in practice, a SF always possesses two neighbouring $\mathrm{X}$-points separated by a finite distance. In such a configuration, the primary $\mathrm{X}$-point determines the LCFS while the secondary X-point can be located either in the private flux region (PFR) of the primary separatrix, a case referred to as snowflake plus $(\mathrm{SF}+) 2$ (b) or in the common flux region, a case referred to as snowflake minus (SF-) 2 (c). The proximity of any SF configuration to an exact SF can be characterised by a parameter $\sigma$, defined as the distance between the $\mathrm{X}$-points, $d_{\mathrm{xpt}}$, normalised with the plasma minor radius on the outboard midplane, $a$.

The different SF configurations can be characterised by two main parameters, $\sigma$ and the angle $\theta$, which is defined as the angle between a line connecting the two X-points and a line perpendicular to a line connecting the primary X-point and the magnetic axis (Figure 2(d)) [12. The $\sigma$ parameter defines the proximity of the divertor configuration to an exact SF, whereas the $\theta$ parameter defines the transition between the $\mathrm{SF}+$ and $\mathrm{SF}$ - . The range $60^{\circ} \lesssim \theta \lesssim 120^{\circ}$ corresponds to a 
(a)

\section{Conventional Single Null (SN)}

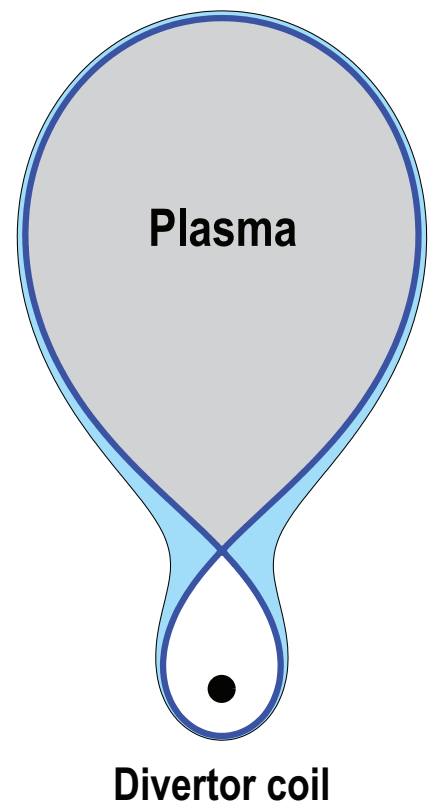

(b)

\section{Snowflake (SF)}

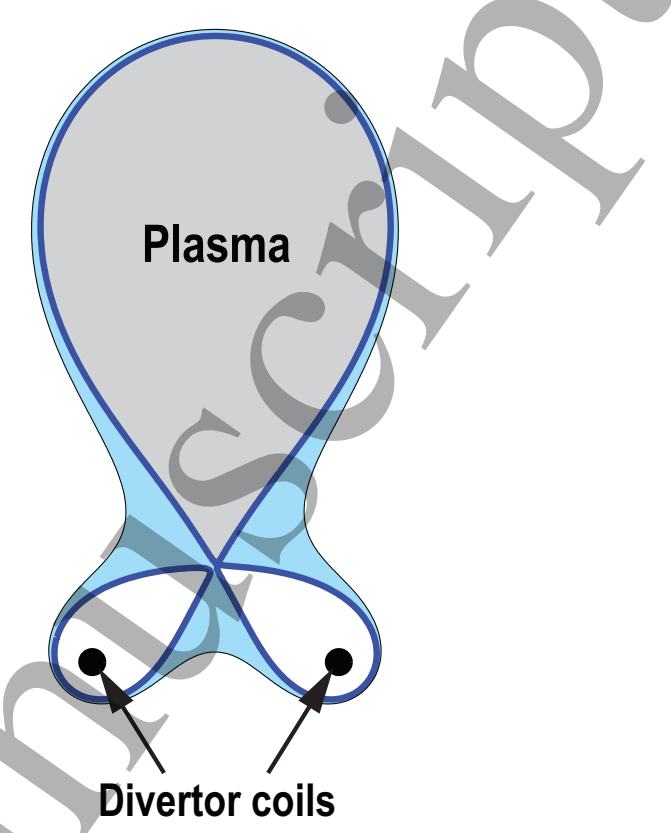

Figure 1: Schematic representations of (a) a conventional single-null and (b) a snowflake divertor.

$\mathrm{SF}+$, while the range defined by $\theta \gtrsim 120^{\circ}$ or $\theta \lesssim 60^{\circ}$ denotes a SF- configuration. Due to the location of the secondary $\mathrm{X}$-point with respect to the primary one, a SF- with $\theta \gtrsim 120^{\circ}$ is referred to as (high field side) HFS SF- whereas a SFwith $\theta \lesssim 60^{\circ}$ is referred to as (low field side) LFS SF-. Since these definitions are based on the expansion of the poloidal flux function near the primary X-point the transition between $\mathrm{SF}+$ and $\mathrm{SF}$ - in practice is not exactly at $60^{\circ}$ and $120^{\circ}$. The SF divertor configuration was first demonstrated experimentally in the TCV tokamak [13, 14]. The configuration has also now been obtained in the NSTX spherical torus [15, 16] and DIII-D tokamak [17].

The small value of $B_{\theta}$ determines several geometric properties of the SF divertor that may be advantageous with respect to reducing the peak and total heat flux to the wall. A large region of low poloidal magnetic field in the vicinity of the null point and a longer connection length are the most relevant geometrical quantities for a SF divertor. The flux expansion in the vicinity of a null-point is enhanced greatly, since the distance between the flux surfaces depends inversely on the magnitude of the poloidal field. An enhanced flux expansion at the target also corresponds to a larger wetted area. A second closely related property is the divertor volume, which increases linearly with the flux expansion. A larger divertor volume is usually associated with larger radiative losses and a greater energy transfer to non-field-directed neutrals. The configuration not only aims at increasing the losses by radiation and neutrals but also for potential SOL 

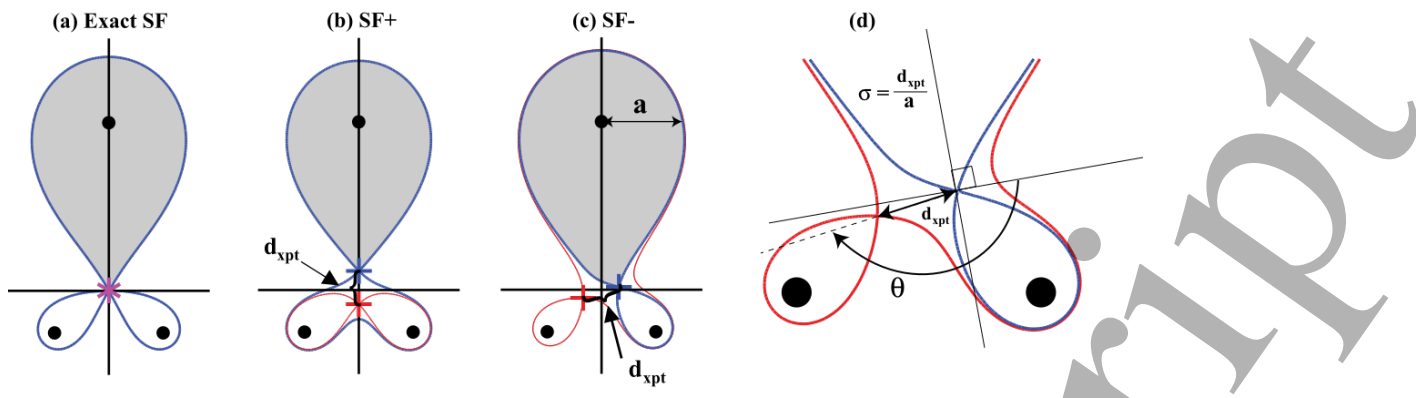

Figure 2: Schematic of different SF configurations: (a) An exact SF, (b) a snowflake plus,(c) a snowflake minus configuration and (d) representation of the $\sigma$ and $\theta$ parameter for a snowflake plasma configuration. The blue and red crosses represent the primary and secondary X-points, respectively, and the black circles represent the plasma magnetic axis and representative divertor coils.

broadening due to enhanced mixing in the null point region [18

The SF plasma configuration is a strong candidate for solving the heat flux problem in future tokamak devices. However, the magnetic configuration of the $\mathrm{SF}$ is highly sensitive to changes in currents within the plasma and external coils and therefore requires complex magnetic control. Thus, an active and precise control of the magnetic configuration is of utmost importance. A real-time snowflake divertor detection algorithm and controller has been successfully implemented in the DIII-D tokamak [19, 20]. EAST tokamak has also successfully demonstrated the real-time shape control of configurations with two magnetic nulls in the divertor [21]. In comparison with the previous published work, the novel generalised plasma position and shape controller developed for the TCV tokamak [22] includes the ability to provide simultaneous control of the position, shape, divertor leg and X-points for the SF plasma configurations and also allows ordering of the controlled variables from the most easily to the least easily controlled, while respecting the hardware limits on the poloidal field coil currents. These unique controller features helps to provide a reliable control of SF equilibria, particularly with closely spaced X-points. However, it must be noted that performance of the new controller relies heavily on the choice of the control parameters. The set of control parameters are chosen based on a trade-off between the level of importance of controlling various plasma quantities and the limits on the poloidal field coil currents. The new controller provides a good tracking of the $\sigma$ parameter with a root mean square deviation (RMSD) of 0.07 in comparison to the TCV analogue controller with a RMSD of 0.15 . Furthermore, it also results in a smaller steady state error in $\sigma$ of 0.13 vs 0.19 for the legacy controller. The performance of the new controller appears less satisfactory for the $\theta$ parameter when $\sigma<0.3$, with a RMSD of $76^{\circ}$ vs $42^{\circ}$ for the analogue controller. This is to be expected as $\theta$ becomes ill-defined as $\sigma$ approaches zero.

The remainder of this paper is structured as follows. Section 2 describes the generalised plasma position and shape controller [22], and introduces the 
reference SF plasma configuration scenario to characterise the performance of the controller. Section 3 describes the design and optimisation of various control parameters of the controller for the SF plasma configuration. Section 4 reports on the controller performance and comparison with that of the legacy TCV analogue controller [23, 24, 25]. A summary of the main experimental results and brief discussion is provided in Section 5 .

\section{Generalized plasma position and shape controller}

Figure 3: Simplified block diagram representation of plasma shape and position control algorithm.

Figure 3 shows the simplified block diagram for the generalised plasma position and shape controller algorithm. A non-diagonal generalised plant $P_{g}$ is defined with poloidal field coil currents as inputs and plasma position and shape variables as outputs; the non-diagonal nature of the plant implies that a change in an input would affect all its outputs [22. Consequently, a compensator design $P_{g}^{+}$, pseudo-inverse of the static (DC) gain of the generalised plant, is derived, provided that $P_{g}$ has a full row (output rank), to counteract the interaction of the plant. The result is a 'newly' shaped plant, $G_{s}=P_{g} P_{g}^{+}$, which is nearly diagonal and easier to control than the original plant $P_{g}$. A diagonal proportional and integral controller $K_{s}$ is utilised, with diagonal gain matrices $K_{s p}$ and $K_{s i}$ to be designed. The control law takes the form of Equation 1, where $\vec{u}$ is the control signal (the vector of 16 poloidal field coil current correction) and $\vec{e}=(\vec{r}-\vec{z})$ is the error defined as the difference between the reference $\vec{r}$ and the controlled variables $\vec{z}$.

$$
\begin{gathered}
\vec{u}=K(s) \vec{e} \\
K(s)=K_{s p}\left(1+\frac{K_{s i}}{s}\right)
\end{gathered}
$$

Figure 4 shows the schematic block diagram for the determination of a generalised plant $P_{g}[22$. A plant $P$ is defined, which accepts poloidal field coil currents 


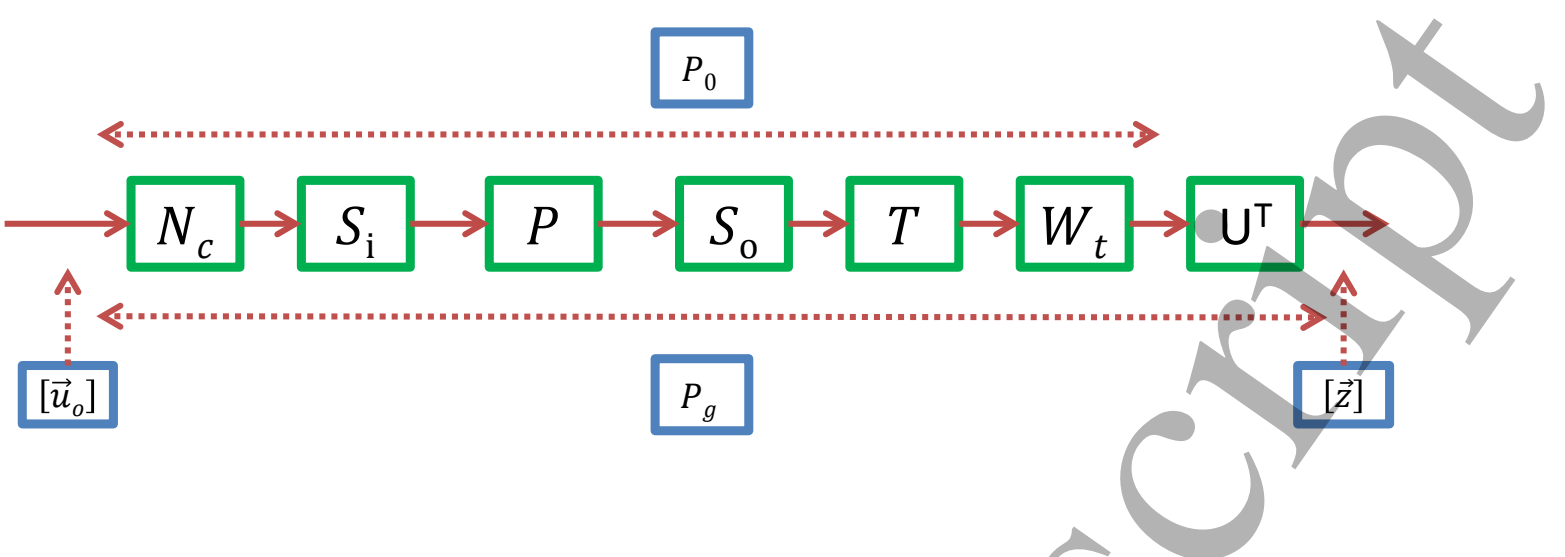

Figure 4: Schematic block diagram for the generalised plant.

as inputs and outputs the poloidal flux $\overrightarrow{\psi_{b}}$ at the control points on the plasma boundary and divertor leg, and poloidal magnetic field $\overrightarrow{B_{b}}$ at the X-points. In particular, the issue of vertical stability, i.e., the growth of the fast axisymmetric mode, is handled by a separate system (namely, the vertical stability control loop in the legacy hybrid control system) and is assumed to be entirely decoupled from the shape-control problem. Neglecting the dynamics and linearising around an equilibrium point, the plant $P$ with $\delta \vec{I}_{P F}$ as inputs and $\delta \vec{y}$ as outputs is given by Equation 2 . Here, $G_{b}$ is the Green's function matrix such that $\delta \overrightarrow{\psi_{b}}=G_{b} \delta \vec{I}_{P F}$, where $\delta \vec{\psi}_{b}$ is the poloidal flux at the control points on the plasma boundary, $G_{d}$ is the Green's function matrix such that $\delta \vec{\psi}_{d}=G_{d} \delta \vec{I}_{P F}$, where $\delta \vec{\psi}_{d}$ the poloidal flux at the control points on the divertor leg, $G_{B_{p}}$ is the Green's function matrix such that $\delta \overrightarrow{B_{p}}=G_{B_{p}} \delta \vec{I}_{P F}$, where $\delta \overrightarrow{B_{p}}$ is the poloidal magnetic field ( $r$ and $z$ compoments) at the X-points. $M_{b}$ is the near-diagonal matrix that transforms absolute poloidal fluxes to flux differences between control points and, similarly, $M_{d}$ is the matrix that transforms absolute fluxes on divertor-leg points to flux differences with respect to the $\mathrm{X}$-point.

$$
P=\left(\begin{array}{c}
M_{b} G_{b} \\
M_{d} G_{d} \\
G_{B_{p}}
\end{array}\right)
$$

The control algorithm includes a constraint, which guarantees that the average addition of poloidal flux at the control points is zero, to prevent interference with the plasma current control. To achieve this, a null space basis $N_{c}$ is determined such that $\left[\mathbf{1}^{T} G_{b}\right] N_{c} u_{o}=0 \forall u_{o} \in \mathbb{R}^{n_{c}-1}$, where $n_{c}$ is the number of poloidal field coils. Thus, the poloidal field coil currents resulting in zero average addition of poloidal flux at the control points is given as, $\delta \vec{I}_{P F}=N_{c} u_{o}$. An input scaling matrix $S_{i}$ ensures common units for the poloidal field coil currents (kA in practice) and an output scaling matrix $S_{o}$ scales the plant outputs to dimensionless quantities that are roughly comparable in magnitude.

A crucial element in the controller formalism is the conversion of the dimen- 


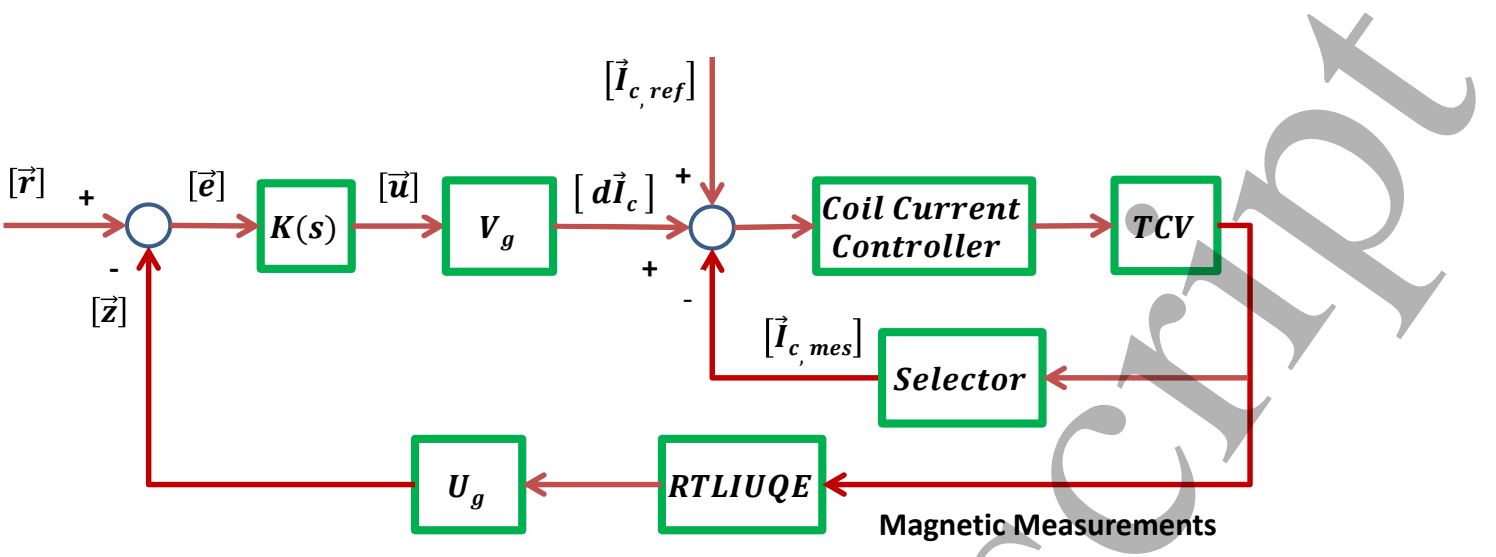

Figure 5: Schematic block diagram of the generalised plasma position and shape controller along with the TCV coil current controller.

sionless plant outputs to physically meaningful estimators or plasma quantities. This operation is performed by designing a matrix, $T$ given by Equation 3 , where $\vec{\psi}_{Z}$ and $\vec{\psi}_{R}$ are the vectors representing the change in the controlled poloidal flux differences due to vertical and radial displacement at the control points on the plasma boundary. A projection of the dimensionless plant outputs onto the vectors corresponding to a vertical and a radial displacement ensures that the first two entries are estimates of the radial/vertical position.

Finally, the estimators are weighted with/the help of a weight matrix $W_{t}$, which provides a means to prioritise the various plasma quantities based on their level of importance for a given plasma configuration.

$$
T=\left(\begin{array}{ccc}
\vec{\psi}_{Z}^{T} & 0 & 0 \\
\vec{\psi}_{R}^{T} & 0 & 0 \\
I & 0 & 0 \\
0 & I & 0 \\
0 & 0 & I
\end{array}\right), \vec{\psi}_{Z}=\frac{M_{b}(\overrightarrow{d \psi} / d Z)}{\left\|M_{b}(\overrightarrow{d \vec{\psi}} / d Z)\right\|_{2}}, \vec{\psi}_{R}=\frac{M_{b}(\overrightarrow{d \psi} / d R)}{\left\|M_{b}(\overrightarrow{d \psi} / d R)\right\|_{2}}
$$

A new plant $P_{o}=W_{t} T S_{o} P S_{i} N_{c}$ is thus finally defined. The next step is to perform a singular value decomposition (SVD) of $P_{o}=U \Sigma V^{T}$, which provides a matrix $V$, representing a set of orthogonal vectors in the admissible input coil current space and $U$, the output directions. This organises the controlled variables into large and small singular values represented by Equation 4, and as a result provides the freedom of limiting the control to the $n_{1}$ largest singular values of $\Sigma_{1}$, obtained by projecting the weighted errors on the output space of these singular values, $U_{1}$.

$$
P_{o}=\left(\begin{array}{ll}
U_{1} & U_{2}
\end{array}\right)\left(\begin{array}{ccc}
\Sigma_{1} & 0 & 0 \\
0 & \Sigma_{2} & 0
\end{array}\right)\left(\begin{array}{c}
V_{1}^{T} \\
V_{2}^{T} \\
V_{3}^{T}
\end{array}\right)
$$


The generalised plant $P_{g}$ is then defined as $P_{g}=U^{1} P_{o}$. The controller for the original plant $P$ is thus represented by Equation 5 .

$$
C_{p}(s)=S_{i} N_{c} P_{g}^{+} K(s)
$$

(a) $\# 55740, \mathrm{I}_{\mathrm{p}}=169(\mathrm{kA}), \kappa=1.6, \delta=0.3$

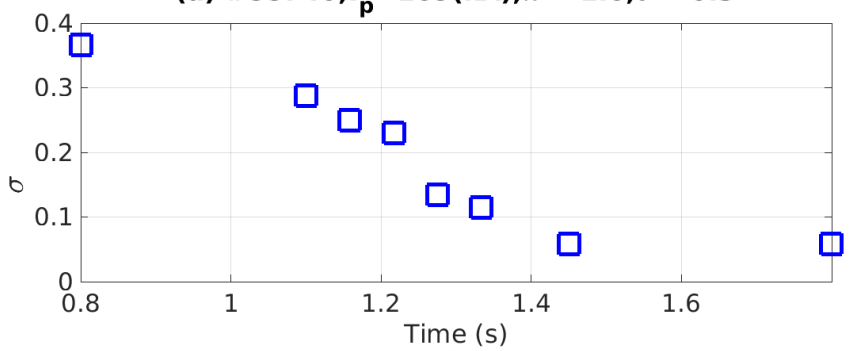

(b)
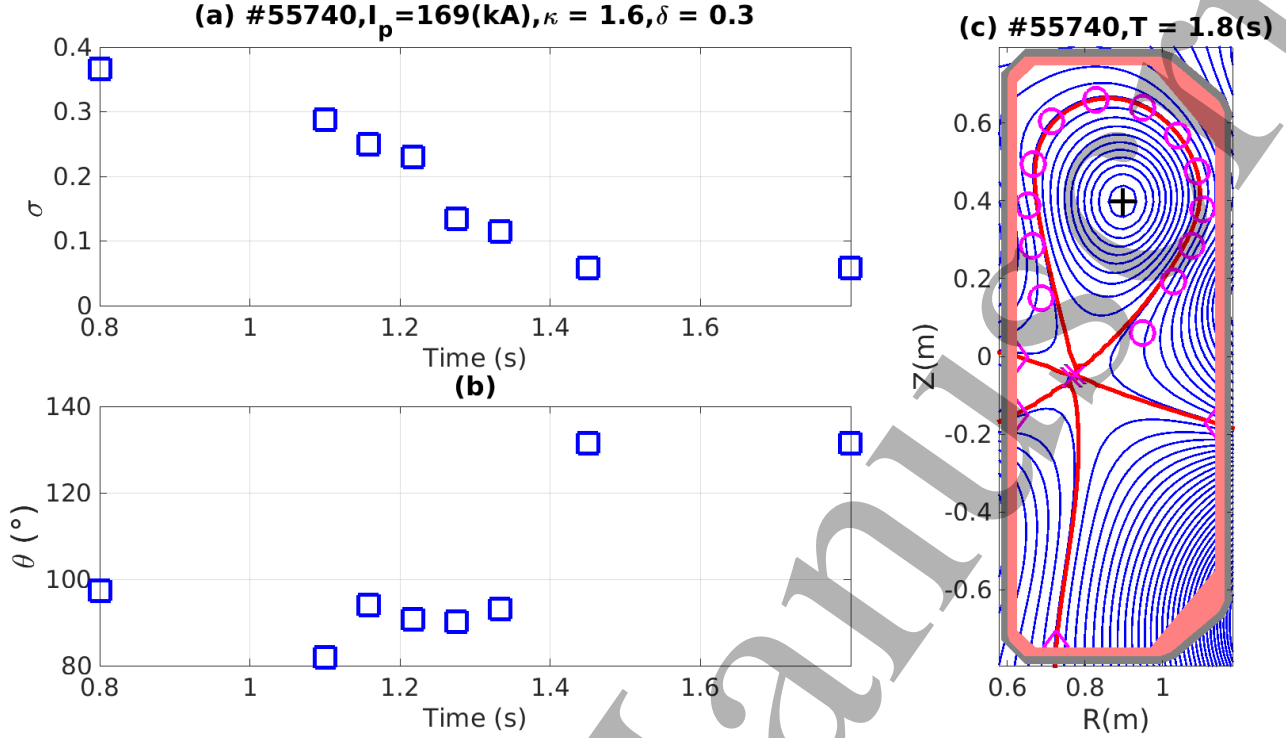

Figure 6: References for (a) $\sigma$ and (b) $\theta$ for a snowflake plasma configuration obtained from FBTE[26].(c) Pre-programmed plasma boundary (magenta circles), location of the strike points (magenta diamonds) and of the X-points (magenta crosses) in MGAMS [27]; poloidal flux map (blue curves) and separatrix (red curves) and location of the magnetic axis (black cross) obtained from the free boundary equilibrium code (FBTE) at a given time instance.

The real-time version of the Grad-Shafranov equilibrium reconstruction code LIUQE [28], RTLIUQE [29], with a sub-ms cycle time $(0.4 \mathrm{~ms})$ provides an estimate of the poloidal flux and magnetic field at the control points. Figure 5 shows the block diagram representing the implementation of the controller with the TCV coil current controller and RTLIUQE. The controller design includes a pre-multiplier matrix $U_{g}=U^{T} W_{t} T S_{o}$ and a post-multiplier matrix $V_{g}=S_{i} N_{c} P_{g}^{+}$: the latter translates the RTLIUQE outputs to controlled variables, $\vec{z}$, while the former provides the actuator combinations for controlling the controlled variables. The reference signals are defined as zeros, since the controlled variables themselves already represent errors in fluxes/fields w.r.t. their desired values. The error signals are fed in to a proportional and integral controller $K(s)$ defined by Equation 1. Detailed information on the controller design can be found in 22 .

RTLIUQE solves for the Grad-Shafranov equation with a least-squares approach that yields the best fit to the magnetic measurements using parametric pressure and current profiles derived partly from measurements and partly from 
modeling or assumptions. The controller formalism assumes, of course, that the estimates of the magnetic fields and poloidal fluxes provided by RTLIUQE are accurate. The performance of the controller must be judged entirely on its ability to create shapes that RTLIUQE will reconstruct. Ultimately, however, the performance of any shape controller will be limited by the accuracy of the shape reconstruction itself. The discussion of the accuracy of RTLIUQE is outside the scope of this paper.

Figure 6] shows the reference Ohmic SF plasma configuration scenario that is used to characterise the performance of the generalised plasma position and shape controller. The scenario consists of a scan of the $\sigma$ parameter for the snowflake plasma configuration and was developed in FBTE/MGAMS [26, 27], the suite of software tools used routinely on TCV to determine the poloidal coil currents and the feedback parameters for a given plasma configuration. The non-overlap of the control points representing the plasma boundary defined in MGAMS with the primary separatrix obtained from FBTE is shown in Figure 6(c). The reason for the non-overlap of the FBTE solution can be found in [26]. The primary control objective of the generalised plasma position and shape controller is to provide a good tracking and zero steady state error of the $\sigma$ parameter for the snowflake plasma configuration. However, at low values of the $\sigma$ parameter, the $\theta$ parameter becomes increasingly sensitive and its controllability decreases accordingly. In addition, the new controller also aims to provide simultaneously, good control of plasma position, shape and divertor leg by controlling the poloidal fluxes at the control points prescribed in MGAMs.

\section{Design and optimisation of control parameters for the SF plasma configuration.}

The primary focus of this work is to achieve reliable control of SF configurations with very low sigma. The two main parameters for tuning the controller performance to ensure good tracking and steady state performance of the $\sigma$ parameter with respect to the given reference scenario are as follows,

- Weight matrix, $W_{t}$, that determines the level of importance of various plasma quantities for a given configuration.

- The number of actively controlled variables, $\vec{z}$, that determines the control of the plasma quantities.

\subsection{Optimisation of the weight matrix}

Inherent to the design of the generalised plasma position and control algorithm is its ability to preferentially weight different plasma quantities. In particular, the position control is ensured by the controller by weighting the vertical and radial position of the plasma such that the first two controlled variables, $\vec{z}$, 
always correspond to vertical and radial control. The first two columns of the post-multiplier matrix, $V_{g}$, represent the coil actuation directions corresponding to the two controlled variables and produce net radial and vertical magnetic fields in the plasma cross-section, respectively, to control the plasma position. A detailed explanation can be found in [22].
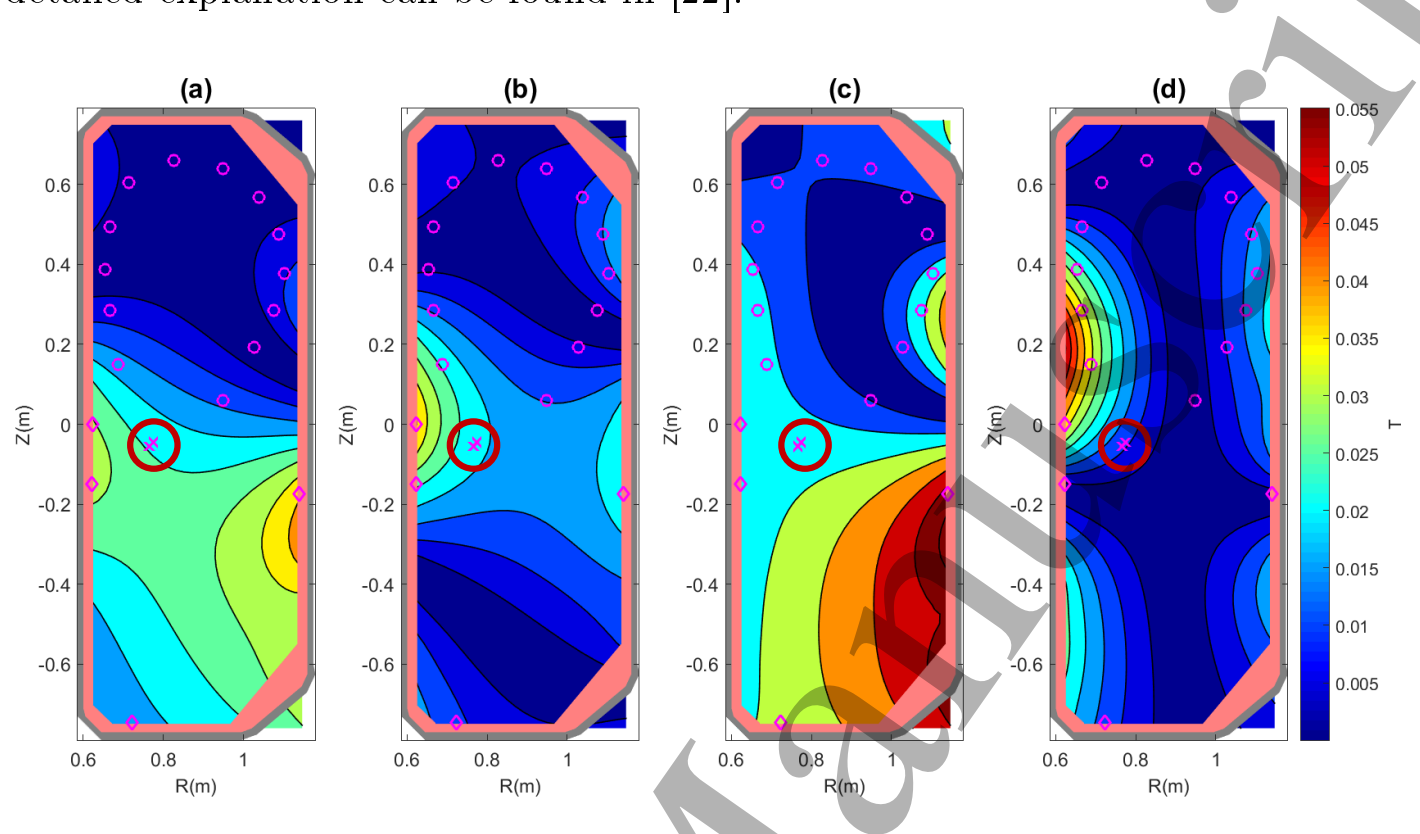

Figure 7: Vertical and radial B-field isoflux surfaces generated by the two sets of poloidal field coil combinations at the location of the X-points (solid red circle) for a SF plasma configuration associated with controlled variable 3 ((a) and (c)) and $4((\mathrm{~b})$ and $(\mathrm{d}))$ with $((\mathrm{a})$ and $(\mathrm{b}))$ and without $((\mathrm{c})$ and $(\mathrm{d}))$ preferential weighting. Pre-programmed plasma boundary (magenta circles), location of the strike points (magenta diamonds) and of the X-points (magenta crosses) from MGAMS [27].

Then, in a scenario with a given set of actively controlled variables, it is possible to choose a suitable weighting on the remaining estimators, e.g. the first moments of the plasma shape, the positions of the strike points and the poloidal magnetic field at the X-points, such that the remaining controlled variables correspond to the above mentioned plasma quantities. Two different sets of $W_{t}$ matrix were chosen, one associated with a higher weighting on the poloidal magnetic field at the X-points, defined as the radial and vertical components of the poloidal magnetic field at the two X-points and the other without any preferential weighting for the plasma quantities. Figures $7(\mathrm{a})$ and (b) shows the calculated isoflux surfaces of vertical and radial magnetic field associated with preferential weighting on the poloidal magnetic field at the X-points for the controlled variables 3 and 4 , respectively. This set of actuator results in net radial and vertical magnetic fields at the X-point location. In comparison, Figures 7 (c) and (d) display the vertical and radial isoflux surfaces associated with variables 3 and 4, respectively, in the case with no preferential weighting. The set of ac- 
tuators associated to the controlled variables 3 and 4 with preferential weighting (Figures $7(\mathrm{a}-\mathrm{b})$ ) produces a poloidal magnetic field peaking nearer the X-points in comparison to the actuator set associated with natural weighting (Figures $7(\mathrm{c}-\mathrm{d}))$.

In order to experimentally confirm the effect of the off-line weighting on the plasma quantities for the SF plasma configuration, plasma discharges based on the reference formulated in Figure 6 were performed with the above sets of $W_{t}$. Figure 8 shows the comparison between a plasma discharge with a higher weighting associated with the poloidal magnetic field at the X-point estimator (55740) and a plasma discharge without any preferential weighting on any nonpositional plasma estimators (55823). Figure 8(a) shows a reduction in the norms of the error on the active set of controlled variables, $\vec{e}$, defined in Equation 1 with the activation of the controller in both discharges. The sum of the norm of the radial and vertical components of the magnetic field at the two X-points are smaller in absolute value in discharge 55740, associated with enhanced weighting on these parameters, than in discharge 55823. (Figures 8(c)) and 8(d)). Figure 8 (b) shows a smaller absolute value of the shape estimator, defined as the norm of the difference between the poloidal flux at the primary X-point and the control points defining the remainder of plasma boundary, in discharge 55823 associated with no preferential weighting, than in discharge 55740. The disturbance in the norm of the error for discharge 55823 in Figure 8(a-d) is a result of the perturbation in the poloidal flux distribution introduced due to a step change in one of the poloidal field coil currents upon switching polarity.

\subsection{Optimisation of the actively controlled variables}

In principle, we dispose of additional degrees of freedom to improve the performance further; namely, with a fixed weighting on the poloidal magnetic field control at the X-points, a larger set of actively $\vec{z}$ can be chosen in order to improve the controller performance, at the expense of a higher demand on the poloidal field coil currents. A series of SF discharges were carried out in order to understand the effect of the number of active controlled variables on the various plasma estimators and on the poloidal field coil currents. The same weighting used for the $\mathrm{X}$-point control in discharge 55740 (discussed in Section 3.1) was kept throughout the scan.

Attempting to increase the number of controlled variables beyond 8 resulted in an over-demand on the coil currents and violation of one of the constraints imposed on the combination of poloidal field coil currents to limit mechanical stresses. As a result, the machine protection interlock system of the TCV tokamak stopped the plasma discharge. Increasing the amount of controlled variables would require imposing these coil current constraints in real-time, and development of associated MIMO anti-windup schemes to treat the saturations in the multi-dimensional coil current input space. This is beyond the scope of the present paper. However, for the purpose of comparison, a SF plasma discharge 
(a) Actively controlled variable errors

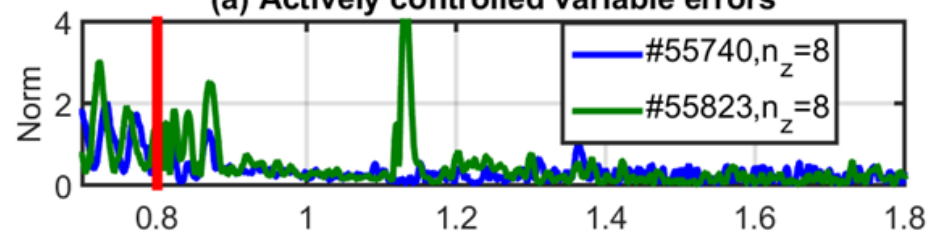

(b) Shape

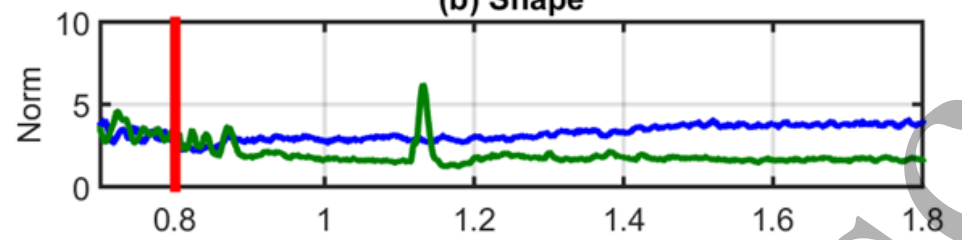

(c) Radial field X-points

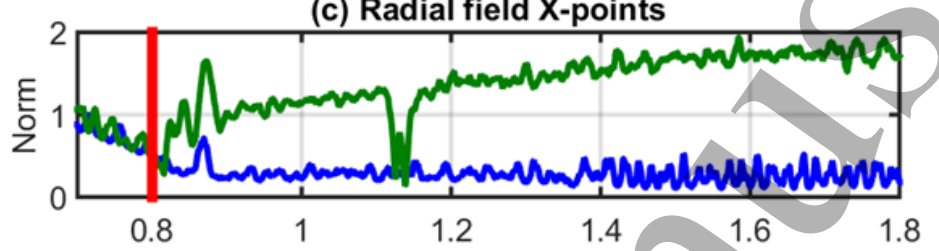

(d) Vertical field X-points

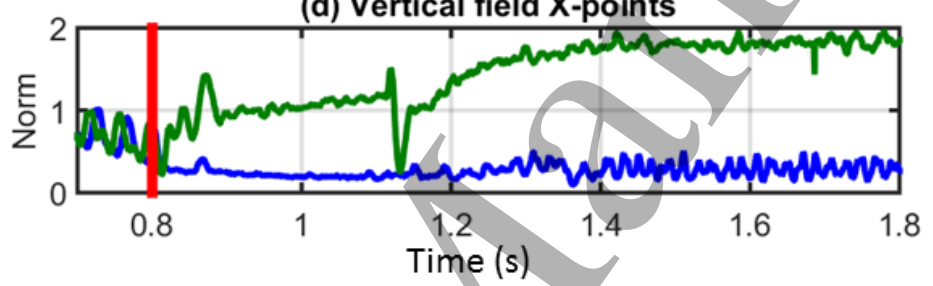

Figure 8: Comparison of controller performance on a SF plasma configuration with (55740) and without (55823) preferential weighting for controlling the poloidal magnetic field at the X-points for an equal number of actively controlled variables $\left(n_{z}=8\right)$. Time evolution of the norm of the errors (a) on the actively controlled variables, (b) over the variables related to plasma shape, (c) over the radial and (d) vertical magnetic fields at the two X-points. The controller activation time is denoted by the red line.

(55843) with only 4 actively controlled variables was performed, this is shown in Figure 9, in comparison with the reference discharge 55740 with 8 variables. Figure 9(a) shows the reduction in the norm of the controlled variable errors after the controller activation. However, Figures 9(b) shows larger plasma shape errors for discharge 55843. In this discharge the control of the poloidal magnetic field at the two X-points is also worse than in discharge 55740 (Figures 9(c) and $9(\mathrm{~d})$ ). In comparison to the large variations shown in Figure 8 , the discharge 55740 with 8 actively controlled variables provides an incremental improvement in comparison to the discharge 55843 with 4 actively controlled variables. 
(a) Actively controlled variable errors

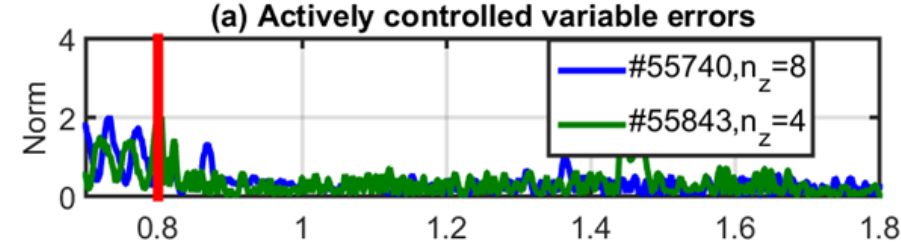

(b) Shape

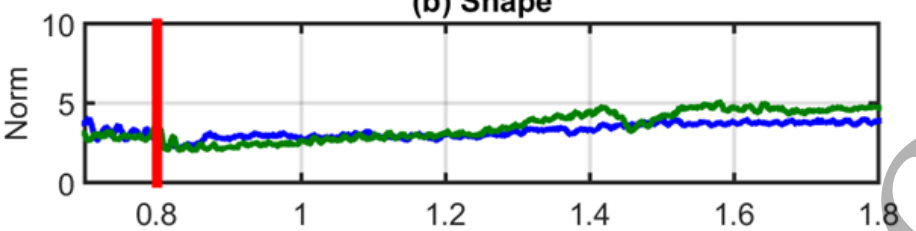

(c) Radial field X-points

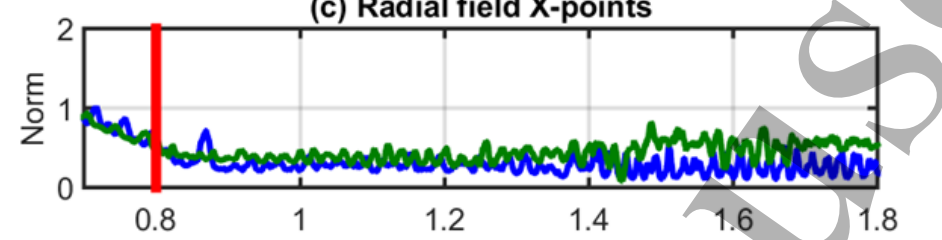

(d) Vertical field X-points

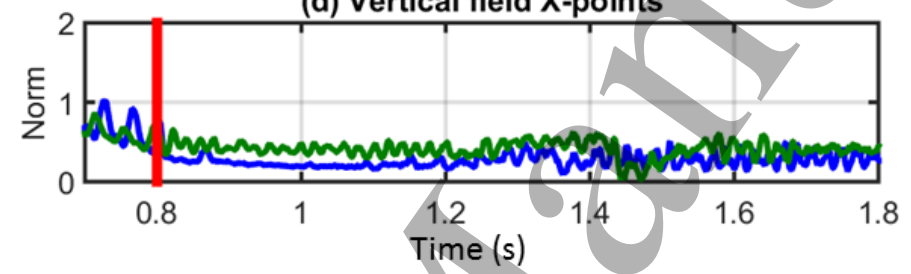

Figure 9: Comparison of controller performance on a SF plasma configuration with $n_{z}=8(55740)$ and $n_{z}=4$ (55843) actively controlled variables with a fixed weighting for controlling the poloidal magnetic field at the X-points. Time evolution of the norm of the errors (a) over all on the active controlled variables, (b) over the variables related to plasma shape, (c) over the radial and (d) vertical magnetic fields at the two $\mathbf{X}$-points. The controller activation time is denoted by the red line.

\section{Controller performance and its comparison with the TCV analogue controller}

The aim of this section is to discuss, for the reference SF plasma configuration defined in Figure 6, the controller performance in comparison with the results achieved using the legacy TCV analogue controller, which merely provides feedforward control for the shape and feedback control of the position using an analog position estimator. These vertical and radial position estimators are constructed directly from magnetic measurements on the vessel wall. All other quantities are controlled only in feedforward, with PF coil currents following references computed by the FBTE, assuming parametric current and pressure profiles. Deviations from the assumptions used in calculating the feedforwards cause departures from the desired shape. This happens in particular as a result of auxiliary heat- 
(a) Snowflake parameter $\sigma$

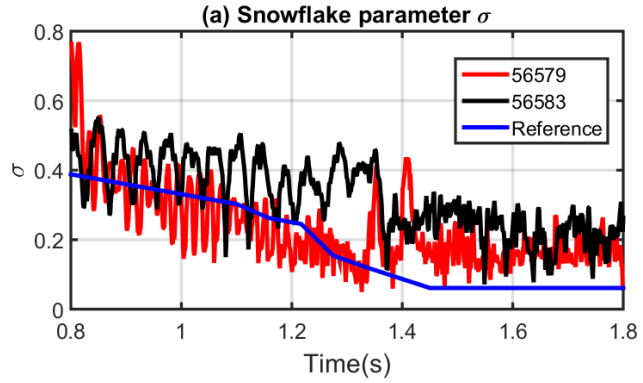

(b) Snowflake parameter $\theta$

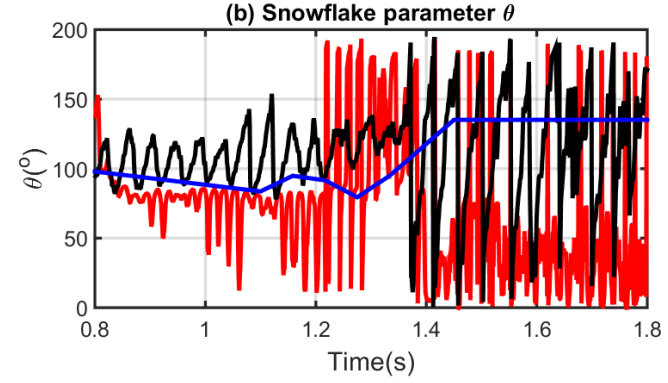

(c) Error:8 Actively controlled variables
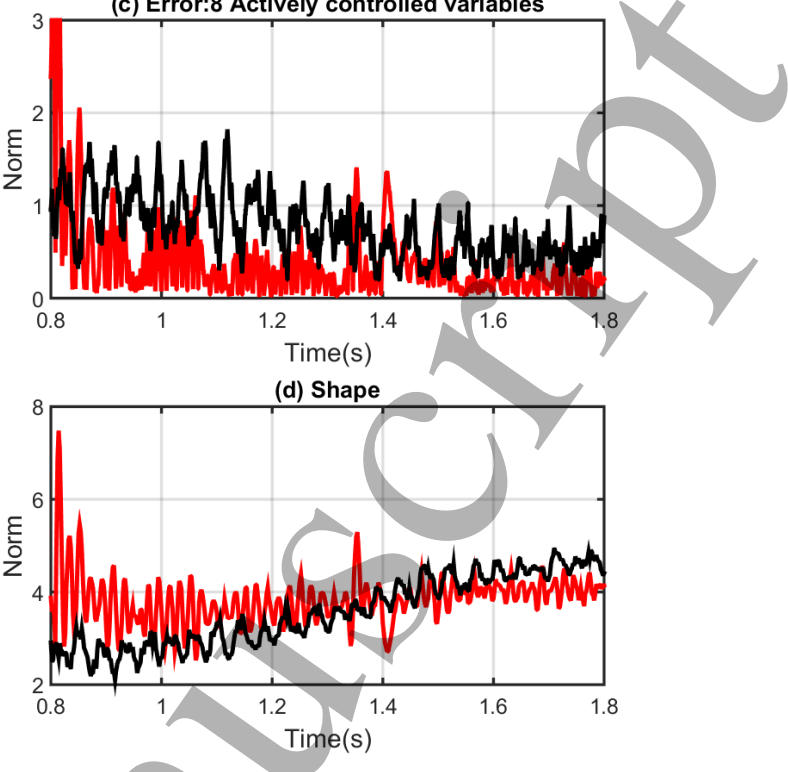

Figure 10: Comparison of the performance of the TCX analogue controller (56583, solid black) and the optimised generalised plasma position and shape controller (56579, solid red) on a SF plasma configuration. Time evolution of the geometrical parameters (a) $\sigma$ and (b) $\theta$; (c) Time evolution of the norm of the errors on the actively controlled variables. (d) Time evolution of the norm of the plasma shape estimator.

ing and current drive altering the current profile. Success in controlling plasma shapes in feedforward mode in TCV has often been achieved by virtue of iterating over multiple discharges.

For comparing the controller performance, we use the reference discharge with optimised controller setup (56579), with 8 active controlled variable and preferential weighting. The same reference was then repeated with the TCV analogue controller emulated on the digital control system of the TCV tokamak (56583). The difference between the performance of the two controllers is evaluated with RTLIUQE. We focus in particular on various properties derived from the equilibrium solver such as e.g. the geometrical parameters for the SF configuration, $\sigma$ and $\theta$.

Figure 10 shows a comparison of the equilibrium properties. Figure 10(a) shows the pre-programmed reference obtained from FBTE and the measurement of the $\sigma$ parameter for the two discharges. The new controller provides better tracking of the $\sigma$ parameter and also leads to lower steady state value $\sigma$ in comparison to the plasma discharge controlled by the legacy TCV controller. However, neither controller is able to achieve the pre-programmed steady state $\sigma$ value. During the phase of constant $\sigma$ operation, a lower steady state error value of 0.13 was obtained for the plasma discharge 56579 associated with the new controller in comparison to a value of 0.19 for 56583 with TCV analogue 
controller. The RMSD between the pre-programmed reference and measurement for $\sigma$ for the discharge 56579 was found to be 0.07 vs 0.15 for the discharge 56583 , for $\sigma<0.1$. The rapid oscillations in the $\theta$ parameter with the new controller, seen in Figure 10(b), are primarily a result of the low value of $\sigma$ reached in this case $(\theta$ becomes undefined for $\sigma=0)$. During the phase of the plasma discharge corresponding to $\sigma<0.3$, the RMSD from the pre-programmed reference of the $\theta$ parameter is lower with a value of $42^{\circ}$ for the discharge 56583 over the value of $76^{\circ}$ for the discharge 56579. Figure 10 (c) illustrates the difference in the norm of the errors, $\vec{e}$, for the two discharges. For the sake of comparison, the norms of $\vec{e}$ are reconstructed for the discharge controlled by the TCV analogue controller. As expected, a smaller norm of the errors is achieved for the discharge 56579 performed with the new controller. Figure $10(\mathrm{~d})$ shows the difference between the norms of the shape estimator for the two discharges. The reference signal for the shape estimators are defined as zeros. For the plasma discharge 56583 performed with the legacy TCV controller, the norm of the/shape estimator increases and diverge from the reference during the plasma discharge, whereas the value of shape estimator remained almost constant for 56579 with a steady state error value of 3.8 .

\section{Discussion and conclusions}

The paper discusses the application of a new generalised plasma position and shape controller to a snowflake plasma configuration, a potential solution to reduce heat loads onto the material surfaces in diverted tokamak plasmas. The paper reports on the experimental investigation of the optimised set of control parameters, primarily the weight matrix $W_{t}$ concerning the control of poloidal magnetic field at the two nearby X-points and the number of actively controlled variables, associated with the control of various plasma quantities and requirements on the poloidal field coil currents. The generalised plasma position and shape controller with an optimised set of control parameters provides good tracking as well as a lower steady state $\sigma$ parameter in comparison to the legacy TCV controller.

The TCV analogue controller has been able to successfully achieve $\sigma$ values ranging from $0.1-1$ and $\theta$ between $10^{\circ}-170^{\circ}$ during various TCV experimental campaigns. The new controller is also able to successfully achieve and track $\sigma$ values ranging from 0.4-0.1 that covers the typical range of values necessary for physics studies on TCV. Rapid oscillations in the $\theta$ parameter ranging from $10^{\circ}$ $170^{\circ}$ denoting transitions between SF plus to SF minus configuration arising at lower values of $\sigma$ observed with both the controllers are not an issue for the TCV physics experiments at these parameters.

In particular, better control of the X-points could conceivably be obtained through a further optimisation of the free control parameters. It is, however, highly encouraging that a low $\sigma$ could be obtained and steadily maintained. 
Whether the value obtained is the ultimate limit for steady-state control or an even lower $\sigma$ is possible remains an open question both from the control standpoint and the SF physics standpoint.

It must be stressed that equilibrium reconstruction is not immune to errors and is particularly difficult for a snowflake plasma in the X-point region. Ultimately, our knowledge of the configuration, and particularly the proximity to the "exact" SF, depends on the accuracy of the reconstruction. While other diagnostics (e.g., cameras) can provide additional information, the precision with which a reconstructed image can identify the X-points is also limited, and typically more limited than the magnetic equilibrium reconstruction itself. However, the crucial result here is the internal consistency of the controller performance i.e., its ability to match the desired reference with a given observer, irrespective of its intrinsic accuracy.

\section{Acknowledgement}

This work was supported in part by the Swiss National Science Foundation This work has been carried out within the framework of the EUROfusion Consortium and has received funding from the Euratom research and training programme 2014-2018 and 2019-2020 under grant agreement No 633053. The views and opinions expressed herein do not necessarily reflect those of the European Commission.

\section{References}

[1] Pitts R A et al. 2009 Physica Scripta T138 014001 ISSN 0031-8949 URL http://stacks.iop.org/1402-4896/2009/i=T138/a=014001

[2] Kotschenreuther M et al. 2007 Physics of Plasmas 14 ISSN 1070664X URL http://dx.doi.org/10.1063/1.2739422

[3] Zohm H et al. 2013 Nuclear Fusion 53073019 ISSN 0029-5515 URL http: //stacks.iop.org/0029-5515/53/i=7/a=073019?key=crossref . d677fdddde2bd2d5b912411cf42615a6

[4] Neilson G H H et al. 2012 Nuclear Fusion 52047001 ISSN 00295515 URL http://stacks.iop.org/0029-5515/52/i=4/a=047001?key= crossref.b606128ba1453c9ad55f5b1acb347b09

[5] Rieth M et al. 2013 Recent progress in research on tungsten materials for nuclear fusion applications in Europe URL http://www.sciencedirect. $\mathrm{com} / \mathrm{science/article/pii/S0022311512004278}$

[6] Kallenbach A et al. 2013 Plasma Physics and Controlled Fusion 55124041 ISSN 0741-3335 URL http://iopscience.iop.org/0741-3335/55/12/ 124041/article/ 
[7] Ryutov D D 2007 Physics of Plasmas 14 ISSN 1070664X URL http://dx. doi.org/10.1063/1.2738399

[8] Ryutov D D et al. 2008 Physics of Plasmas 15 ISSN 1070664X URL http: //dx.doi.org/10.1063/1.2967900

[9] Kukushkin A S et al. 2005 Nuclear Fusion 45 608-616 ISSN 0029-5515 URL http://stacks . iop. org/0029-5515/45/i=7/a=008?key= crossref.acf4e3fbadb57d6d36567326d98e7bea

[10] Petrie T W et al. 1997 Nuclear Fusion 37643 ISSN 0029-5515 URL http: //iopscience.iop.org/0029-5515/37/5/I07

[11] Valanju P M et al. 2009 Super-X divertors and high power density fusion devices Physics of Plasmas vol 16 ISBN 1070-664X ISSN 1070664X URL http://dx.doi.org/10.1063/1.3110984

[12] Ryutov D D et al. 2010 Plasma Physics and Controlled Fusion 52 ISSN 07413335

[13] Piras F et al. 2009 Plasma Physics and Controlled Fusion 51055009 ISSN 0741-3335 URL http://stacks/iop.org/0741-3335/51/i=5/a=055009? key=crossref. a281e046d21242eba91cedfc60587859

[14] Reimerdes H et al. 2013 Plasma Physics and Controlled Fusion 55124027 ISSN 0741-3335 URL http://stacks.iop.org/0741-3335/55/i=12/a= 124027?key=crossref .bc4a3adf 8 acbb82cfb7f392553940e52

[15] Soukhanovskii V A et al. 2012 Physics of Plasmas 19 ISSN 1070664X URL http://dx.doi.org/10.1063/1.4737117

[16] Vail P J et al. 2019 Plasma Physics and Controlled Fusion 61 ISSN 13616587

[17] Soukhanovskii V A et al. 2015 Journal of Nuclear Materials 463 1191-1195 ISSN 00223115.URL http://www.sciencedirect.com/science/article/ pii/S0022311514009945

[18] Ryutov D D et al. 2014 The 'churning mode' of plasma convection in the tokamak divertor region

[19] Kolemen E et al. 2015 Journal of Nuclear Materials 463 1186-1190 ISSN 00223115 URL http://dx.doi.org/10.1016/j.jnucmat.2014.11.099

[20] Kolemen E et al. 2018 Nuclear Fusion 58 ISSN 17414326

[21] Yuan Q P et al. 201846 1356-1360

[22] Anand $\mathrm{H}$ et al. 2017 Accepted for publication in Nuclear Fusion 57 ISSN 17414326 
[23] Le H B et al. 2014 Fusion Engineering and Design 89 155-164 ISSN 09203796 URL http://www.sciencedirect.com/science/article/pii/ S0920379613006996

[24] Felici F et al. 2014 Fusion Engineering and Design 89 166-176/ISSN 09203796

[25] Anand H et al. 2017 Distributed digital real-time control system for the TCV tokamak and its applications Nuclear Fusion vol 57 (IOP Publishing) p 056005 ISSN 17414326 URL http://stacks.iop.org/0029-5515/57/i= $5 / a=056005$

[26] Hofmann F 1988 Computer Physics Communications 48 207-221 ISSN 00104655 URL //www.sciencedirect.com/science/article/pii/ 0010465588900410

[27] Hofmann F et al. 1995 Plasma Shape Control in TCV using MGAMS 22rd EPS Conference vol. 19C (Bournemouth) pp II-101

[28] Hofmann F et al. 1988 Nuclear Fusion 28 519-522 ISSN 00295515 URL http: //stacks.iop.org/0029-5515/28/i=3/a=015?key=crossref . 59b80c614f2043fbd9bd234e5a94265d

[29] Moret J M et al. 2015 Fusion Engineering and Design 91 1-15 ISSN 09203796 URL //www.sciencedirect.com/science/article/pii/ S0920379614005973 